\title{
Structure Of Demand And Equilibrium In Oligopolistic Models With Piecewise Quadratic Cost Functions
}

\author{
Natalia I. Kalashnikova (E-mail: nkalash@fcfm.uanl.mx), University of Nuevo Leon, Mexico
}

\begin{abstract}
In this paper, we consider an oligopolistic model with conjectures concerning the price variations depending upon the agents' production increase or increase. By introducing the notion of an interior stable equilibrium, we analyze the behavior of groups of consumers with different consumption abilities. The proposed techniques allow us to make a qualitative description of dependence of the market price upon the active demand component.
\end{abstract}

\section{Introduction}

W

hen studying the oligopolistic market in the framework of the classical models [1] - [3], besides the questions of an equilibrium existence and its computation, a lot of attention is usually paid to comparing the latter with the equilibrium in the perfect competition model. Recently [4] - [5], both models were included in a united class of oligopoly models where the degree of influence on the whole situation by each agent is modeled by some special parameters (influence coefficients). If we set these influence coefficients in an exogenous way, then the demand structure does not affect the production regime, i.e. the dependence of output values upon the market price. Although a deformation of the demand curve leads to a variation of equilibrium, the possible modeling of the deformation influence in such models is limited. For example, it is difficult to trace a rapid (or even jump-like) variation of the market price, a stable price under the demand growth (within certain limits), and a series of other phenomena.

However, if one does not consider the influence coefficients as given beforehand but includes them in the definition of the equilibrium itself, together with the equilibrium price and production volumes, one obtains a possibility to simulate the above-mentioned effects. Such a notion of equilibrium was introduced in the paper by V.A. Bulavsky [6]. There, the influence coefficients are determined by a special verification procedure that checks their consistency. The procedure in [6] supposes that each agent can observe the variations of the aggregate production output as a response to his own output variations and thus estimate his influence. The choice of the aggregate production volume as the observed parameter was done due to the fact that the classical Cournot model was taken as the basis of the developed model. Nevertheless, one can assume that it is more natural to observe the market price instead of the aggregate output, and hence estimate each agent's influence upon the price. Such an approach is developed in paper [7], which allowed to relax the conditions imposed on the demand function and keep on in essence only one of them, namely its being non-increasing when the price increases. A technical condition for the latter function of being piecewise smooth is also kept valid.

In such a framework in [7], the equilibrium existence was established, and certain consequences of the demand structure variations were examined. Namely, two types of variations were illustrated. The first one is related to the fall down of consumption ability of one group of consumers with the consumption ability of another group of

Readers with comments or questions are encouraged to contact the author via email. 
consumers kept intact. It turned out that the proposed model predicted three stages of the process: (i) the price going down with the aggregate production output kept constant, (ii) the price going down further with the simultaneous production decreasing and a deficit arising, (iii) and at last, a sharp jump of the price without production growth, and the deficit disappearing on account of the poorer consumers abandoning the market.

Before mentioning the second type of the demand structure variations we insert a certain elucidation. In the classical oligopoly models, the aggregate bargain volume decreases as the price grows up. This fact goes in line with the postulate of the price falling down as the supply increases. However, there exists a dual (in a certain sense) postulate: the price increases as the demand goes up. In order to remove this seeming contradiction, one should make distinction between two kinds of demand: a passive and an active one.

The standard demand function in the classical oligopoly models describes the passive demand: the consumers are waiting for a good to be proposed to them at some price and then decide to buy it or not. However, the active demand does not depend on the price and constitutes the additional component of the aggregate demand. So when the active demand increases the market price should grow up.

It is the growth of the active demand component that is the second type of the demand structure variations examined in [7]. The active component may reflect both an agitating demand and the demand related to some needs outside the model. For example, it could be a demand arising because of some military actions, development of the defense material production, etc. It was shown in [7] that the active demand growing, in the presence of a group of passive consumers, also provides three phases to be observed: (i) the price kept invariable while the production goes up, (ii) the constant price with the constant production volume and hence, a deficit appearing, (iii) and at last, the price jumping up as the market is abandoned by the passive consumers, and only the active demand is satisfied thereafter.

An interesting phenomenon was also mentioned in [7]: with both the first and the second type of the demand structure variations, the process is not invertible completely. For example, if the leap in price is followed by the active demand going down, then the price does not return to the initial level unless the active demand becomes lower than the initial one! Similarly, in the second example, the initial price is not reached unless the poorer group is drawn up to the state of the richer group but not to the poor initial state.

The aim of this article is to relax the conditions under which the latter behavior of the equilibrium price takes place, by permitting the cost functions to be piecewise quadratic, with possible breaks of their second derivatives (but not of their first derivatives).

In the next section, we first describe the mathematical model from [7] and then extend the techniques to the case of piecewise quadratic cost functions. The qualitative analysis of the behavior of the market price depending upon the active demand variations constitutes the last section. The proofs of the results are omitted due to the space restrictions, and they will be published eslewhere.

\section{Model Specification}

Consider $\boldsymbol{n} \geq \mathbf{3}$ producers of a homogeneous good with the cost functions $\boldsymbol{f}_{i}\left(\boldsymbol{q}_{i}\right), \boldsymbol{i}=\mathbf{1}, \ldots, \boldsymbol{n}$, where $\boldsymbol{q}_{i}$ is the output by producer $\boldsymbol{i}$. Consumers demand is described by a demand function $\boldsymbol{G}(\boldsymbol{p})$, whose argument $p$ is the price proposed by the producers. An active demand $\boldsymbol{D}$ is non-negative and does not depend upon the price. The demand function is assumed to be non-negative and non-increasing, and also continuously differentiable everywhere except for a finite number of points in which both the function $G(p)$ and its derivative $G^{\prime}(p)$ may be discontinuous. Because of that, we denote properly by $\boldsymbol{G}(p)$ the demand function's left limit at the point $\boldsymbol{p}$, whereas the right limit we denote by $g(p)$. It is straightforward that $G(p)=g(p)$ at the continuity points while $G(p)>g(p)$ at the discontinuity ones. In the latter case, $G(p)$ can be treated as the potential market capacity, and $g(p)$-- as the lower limit of supply preventing the price growing up. We will reflect the equilibrium between the demand and supply for a given price $\boldsymbol{p}$ by the following pair of inequalities 


$$
g(p)+D \leq \sum_{i=1}^{n} q_{i} \leq G(p)+D .
$$

We collect the above assumptions into the formal assumption below.

A1. The demand function is defined for prices $\boldsymbol{p} \in(\mathbf{0},+\infty)$ being non-increasing and piecewise continuously differentiable one. At each of the finite number of discontinuity points there exist left- and right-hand limits of both the function itself (due to its monotonicity) and its derivative.

Now we pay attention to cost functions which are supposed to be convex, continuously differentiable and having piecewise continuous second derivative. Producer $i$ chooses its output volume $\boldsymbol{q}_{\boldsymbol{i}}$ so as to maximize its profit value $\boldsymbol{p} \cdot \boldsymbol{q}_{i}-\boldsymbol{f}_{\boldsymbol{i}}\left(\boldsymbol{q}_{i}\right), \boldsymbol{q}_{i} \geq \mathbf{0}$ assuming that the choice may affect the price value $\boldsymbol{p}$. The latter assumption could be defined by a conjectured dependence of the price $\boldsymbol{p}$ upon the output value $\boldsymbol{q}_{i}$. If so, the first order maximum condition to describe the equilibrium would have the form

$$
p+q_{i} \frac{\partial p}{\partial q_{i}}-f^{\prime}{ }_{i}\left(q_{i}\right)\left\{\begin{array}{l}
=0, q_{i}>0 \\
\leq 0, q_{i}=0
\end{array}\right.
$$

Thus we see that to describe the agent's behavior, not the dependence of $\boldsymbol{p}$ upon $\boldsymbol{q}_{i}$ itself but its derivative $\frac{\partial p}{\partial q_{i}}=-v_{i}$ is important. We introduce the minus here in order to deal with positive values of $v_{i}$. Of course, the conjectured dependence of $\boldsymbol{p}$ on $\boldsymbol{q}_{\boldsymbol{i}}$ must provide (at least local) concavity of the $\boldsymbol{i}$-th agent's conjectured profit as a function of his output. Otherwise one cannot guarantee the profit to be maximized (but not minimized). As we suppose that the cost functions $\boldsymbol{f}_{\boldsymbol{i}}\left(\boldsymbol{q}_{\boldsymbol{i}}\right)$ are convex, then the concavity of the product $\boldsymbol{p} \cdot \boldsymbol{q}_{\boldsymbol{i}}$ will do. For instance, it is enough to assume the coefficient $\boldsymbol{v}_{\boldsymbol{i}}$ (called from now on as the $\boldsymbol{i}$-th agent's influence coefficient) to be nonnegative and constant. Then the conjectured local dependence of the profit upon the production output $\eta_{i}$ has the form $\left[p-v_{i}\left(\eta_{i}-q_{i}\right)\right] \eta_{i}-f_{i}\left(\eta_{i}\right)$, while the maximum condition at $\eta_{i}=q_{i}$ is provided by the relationships

$$
\left\{\begin{array}{l}
p=v_{i} q_{i}+f^{\prime}{ }_{i}\left(q_{i}\right), q_{i}>0 ; \\
p=f^{\prime}{ }_{i}(0), \quad q_{i}=0
\end{array}\right.
$$

Were the agents' conjectures about the model given exogenously like it was done in [4] - [5], we would allow the values $v_{i}$ to be functions of $\boldsymbol{q}_{i}$ and $\boldsymbol{p}$. However, here we use the approach from the papers [6]- [7] where the conjecture parameters for an equilibrium are determined simultaneously with the price $\boldsymbol{p}$ and the output values $\boldsymbol{q}_{i}$ by a special verification procedure. In the latter case, the influence coefficients are the scalar parameters determined only for the equilibrium. Onward, such an equilibrium is referred to as interior and is described by the parameter set $\left(p, q_{1}, \ldots, q_{n}, v_{1}, \ldots, v_{n}\right)$.

In order to present the verification procedure we need the second notion of equilibrium called exterior (cf. [7]) with parameters $v_{i}$ given exogenously.

Definition 1. The set $\left(\boldsymbol{p}, \boldsymbol{q}_{1}, \ldots, \boldsymbol{q}_{\boldsymbol{n}}\right)$ is called an exterior equilibrium for given influence coefficients $\left(\boldsymbol{v}_{1}, \ldots, v_{n}\right)$, if the market is balanced, i.e. condition (1) is satisfied, and for each $i$ the maximum conditions (2) are valid.

Assumptions concerning the cost functions of the model participants are given below. 
A2. For each $\boldsymbol{i}=\mathbf{1}, \ldots, \boldsymbol{n}$, the function $\boldsymbol{f}_{\boldsymbol{i}}\left(\boldsymbol{q}_{\boldsymbol{i}}\right)$ is defined for $\boldsymbol{q}_{\boldsymbol{i}} \geq \mathbf{0}$ and is piecewise twice continuously differentiable with $f_{i}{ }^{\prime}(\mathbf{0})>\mathbf{0}$ and $\boldsymbol{f}_{i}{ }^{\prime \prime}\left(\boldsymbol{q}_{i}\right)>\mathbf{0}$ for each $\boldsymbol{q}_{i} \geq \mathbf{0}$, except for a finite subset of points.

Example 1. The function defined below, being piecewise quadratic satisfies assumption A2:

$$
f(x)= \begin{cases}\left(x^{2}+2 x\right) / 2, & 0 \leq x \leq 1 \\ \left(2 x^{2}+1\right) / 2, & 1<x \leq 2 \\ \left(x^{2}+4 x-3\right) / 2, & x>2 .\end{cases}
$$

Indeed, it is continuously differentiable over the half-axis $\left[\mathbf{0 , + \infty}\right.$, with $f_{i}{ }^{\prime}(\mathbf{0})=\mathbf{1}$ and is twice continuously differentiable over the intervals $(\mathbf{0 , 1}),(\mathbf{1}, \mathbf{2})$, and $(\mathbf{2},+\infty)$. In the key points $\boldsymbol{x}=\mathbf{0}, \boldsymbol{x}=\mathbf{1}$, and $\boldsymbol{x}=\mathbf{2}$, the function $f(x)$ has only one-side second derivatives which are as follows:

$$
f^{\prime \prime}(0+0)=1, f^{\prime \prime}(1-0)=1 \neq 2=f^{\prime \prime}(1+0), \text { and } f^{\prime \prime}(2-0)=2 \neq 1=f^{\prime \prime}(2+0) .
$$

In what follows we are going to consider only the case when the participants set is constant independently from the values $v_{i}$ of the influence coefficients. To provide this, we make the following assumption.

A3. At the price $\boldsymbol{p}_{\mathbf{0}}=\max _{\mathbf{1} \leq i \leq n}\left\{\boldsymbol{f}^{\prime}{ }_{i}(\mathbf{0})\right\}$ for any $\boldsymbol{i}$ there exists (uniquely, due to A2) an output volume $\boldsymbol{q}_{i}^{\mathbf{0}}$ such that $p_{0}=f^{\prime}{ }_{i}\left(q_{i}^{0}\right)$, moreover, $\sum_{i=1}^{n} q_{i}^{0}<g\left(p_{0}\right)$.

The latter condition guarantees that for any $\boldsymbol{v}_{\boldsymbol{i}}$, conditions (1) and (2) can hold simultaneously only for $\boldsymbol{p}>\boldsymbol{p}_{\mathbf{0}} \geq \boldsymbol{f}^{\prime}{ }_{i}(\mathbf{0})$, and hence only for all $\boldsymbol{q}_{i}$ being strictly positive.

Theorem 1. Under assumptions $\mathbf{A 1}, \mathbf{A 2}$ and $\mathbf{A 3}$, for any $\mathbf{D} \geq \mathbf{0}$ and $\boldsymbol{v}_{\boldsymbol{i}} \geq \mathbf{0}, \boldsymbol{i}=\mathbf{1}, \ldots, \boldsymbol{n}$, there exists uniquely an exterior equilibrium $\left(\boldsymbol{p}, \boldsymbol{q}_{\mathbf{1}}, \ldots, \boldsymbol{q}_{\boldsymbol{n}}\right)$ which depends continuously upon the parameters set $\left(\boldsymbol{D}, \boldsymbol{v}_{\mathbf{1}}, \ldots, \boldsymbol{v}_{\boldsymbol{n}}\right)$. The equilibrium price as a function of these parameters has the left side and right side derivatives by $\boldsymbol{D}$. Moreover, $\boldsymbol{p}>\boldsymbol{p}_{\mathbf{0}}$, and

$$
\begin{aligned}
& p^{\prime}(D+0)=\frac{1}{\sum_{i=1}^{n} \frac{1}{v_{i}+f^{\prime \prime}{ }_{i}\left(q_{i}+0\right)}-G^{\prime}(p+0)} \text {, if } \sum_{i=1}^{n} q_{i}=g(p)+D \text {; } \\
& p^{\prime}(D-0)=\frac{1}{\sum_{i=1}^{n} \frac{1}{v_{i}+f^{\prime \prime}{ }_{i}\left(q_{i}-0\right)}-G^{\prime}(p-0)}, \text { if } \sum_{i=1}^{n} q_{i}=G(p)+D \text {; } \\
& p^{\prime}(D+0)=0, \quad \text { if } \sum_{i=1}^{n} q_{i}>g(p)+D \text {; } \\
& p^{\prime}(D-0)=0, \quad \text { if } \sum_{i=1}^{n} q_{i}<G(p)+D .
\end{aligned}
$$


Let us close the graph of the demand function by connecting the discontinuity points $(p, G(p))$ and $(p, g(p))$ by a vertical line. Then we obtain a line $L$ on the plane $(p, G)$; from now on, we will call it the demand curve, as it comprises all the points $(p, G)$ satisfying the inequalities $g(p) \leq G \leq G(p)$. On the vertical segments of this line it is convenient to set $G^{\prime}(p+0)=-\infty$ if $G>g(p)$, and $G^{\prime}(p-0)=-\infty$ if $G<G(p)$. The exterior equilibrium $\left(p, q_{1}, \ldots, q_{n}\right)$ determines the point $(p, G) \in L$ such that $G=\sum_{i=1}^{n} q_{i}-D$. Under the above assumptions, the formulas of Theorem 1 can be rewritten uniformly as follows:

$$
p^{\prime}(D \pm 0)=\frac{1}{\sum_{i=1}^{n} \frac{1}{v_{i}+f^{\prime \prime}{ }_{i}\left(q_{i} \pm 0\right)}-G^{\prime}(p \pm 0)} .
$$

Therefore, $\quad \boldsymbol{p}^{\prime}(\boldsymbol{D}+\boldsymbol{0}) \neq \boldsymbol{p}^{\prime}(\boldsymbol{D}-\mathbf{0})$ only for the equilibria associated with the corner points of the graph $\boldsymbol{L}$. Besides, if $\boldsymbol{p}^{\prime}(\boldsymbol{D}+\mathbf{0})<\boldsymbol{p}^{\prime}(\boldsymbol{D}-\mathbf{0})$ then the corner point is concave, while it is convex if $\boldsymbol{p}^{\prime}(\boldsymbol{D}+\mathbf{0})>\boldsymbol{p}^{\prime}(\boldsymbol{D}-\mathbf{0})$.

Now we are ready to define an interior equilibrium. To do that, we first describe the procedure of verification of the influence coefficients $\boldsymbol{v}_{\boldsymbol{i}}$ as it was given in [6]--[7]. Assume that we have an exterior equilibrium $\left(\boldsymbol{p}, \boldsymbol{q}_{\mathbf{1}}, \ldots, \boldsymbol{q}_{\boldsymbol{n}}\right)$ that occurred for some $v_{1}, \ldots, v_{n}$ and $D$. One of the producers, say $k$, temporarily changes his behavior by abstaining from maximization of the conjectured profit and making small fluxions around his equilibrium output volume $\boldsymbol{q}_{\boldsymbol{k}}$. In mathematical terms it is tantamount to restricting the model agents to the subset $\boldsymbol{i} \neq \boldsymbol{k}$ with the volume $\boldsymbol{q}_{\boldsymbol{k}}$ subtracted from the active demand. Variation of agent $\boldsymbol{k}$ production output is then reduced to the same absolute value variation (but to the opposite side) of the active demand $\boldsymbol{D}_{\boldsymbol{k}}=\boldsymbol{D}-\boldsymbol{q}_{\boldsymbol{k}}$. If we consider these variations being infinitesimal, we assume that by observing the corresponding variations of the equilibrium price, agent $\boldsymbol{k}$ gets the left-side and right-side derivatives of the equilibrium price with respect to the active demand, i.e. his left-side and right-side influence coefficients.

If the equilibrium point $\left(p, G=\sum_{i=1}^{n} q_{i}\right)$ belongs to the smooth part of the graph $L$ of the demand function, then these derivatives values coinside, and the consistent (verified) influence coefficient also must be equal to this value. However, if $(\boldsymbol{p}, \boldsymbol{G})$ is a corner point, then the coefficient $\boldsymbol{v}_{\boldsymbol{k}}$ is admitted as consistent if it lies between the left-side and right-side derivative values of the equilibrium price. Applying Theorem 1 or, which is the same, formula (3) to calculate the derivatives, one has to remember that agent $\boldsymbol{k}$ is temporarily outside the equilibrium model, hence one has to exclude the addendum with the number $\boldsymbol{i}=\boldsymbol{k}$. Having that in mind, we come to the following criterion.

Consistency Criterion. At an exterior equilibrium $\left(\boldsymbol{p}, \boldsymbol{q}_{1}, \ldots, \boldsymbol{q}_{\boldsymbol{n}}\right)$ the influence coefficient $\boldsymbol{v}_{\boldsymbol{k}}$ is admitted as consistent if there exists a value $\boldsymbol{r}_{\boldsymbol{k}}$ such that

$$
\min \left\{G^{\prime}(p-0), G^{\prime}(p+0)\right\} \leq r_{k} \leq \max \left\{G^{\prime}(p-0), G^{\prime}(p+0)\right\}
$$

and 


$$
\begin{aligned}
& \min \left\{\left(\sum_{i \neq k}\left[v_{i}+f^{\prime \prime}{ }_{i}\left(q_{i}-0\right)\right]^{-1}-r_{k}\right)^{-1},\left(\sum_{i \neq k}\left[v_{i}+f^{\prime \prime}{ }_{i}\left(q_{i}+0\right)\right]^{-1}-r_{k}\right)^{-1}\right\} \leq v_{k} \leq \\
& \leq \max \left\{\left(\sum_{i \neq k}\left[v_{i}+f^{\prime \prime}{ }_{i}\left(q_{i}-0\right)\right]^{-1}-r_{k}\right)^{-1},\left(\sum_{i \neq k}\left[v_{i}+f^{\prime \prime}{ }_{i}\left(q_{i}+0\right)\right]^{-1}-r_{k}\right)^{-1}\right\}
\end{aligned}
$$

where $f^{\prime \prime}{ }_{i}\left(q_{i} \pm 0\right)=\lim _{s \rightarrow q_{i} \pm 0} f^{\prime \prime}{ }_{i}(s)$. Clearly, if $q_{i}$ is a continuity point of the second derivative of each cost function $\boldsymbol{f}_{\boldsymbol{i}}, \boldsymbol{i} \neq \boldsymbol{k}$, then the previous conditions reduce to the following equality:

$$
v_{k}=\frac{1}{\sum_{i \neq k} \frac{1}{v_{i}+f^{\prime \prime}{ }_{i}\left(q_{i}\right)}-r_{k}}
$$

Here the values $G^{\prime}(p-0)$ and $G^{\prime}(p+0)$ are taken at the point $\left(p, G=\sum_{i=1}^{n} q_{i}-D\right)$ of the demand function graph L. If $\boldsymbol{r}_{\boldsymbol{k}}=-\infty$ then $\boldsymbol{v}_{\boldsymbol{k}}=\mathbf{0}$.

Before we define the interior equilibrium we notice that when the equilibrium corresponds to a smooth point on the demand function graph then the numbers $\boldsymbol{r}_{\boldsymbol{k}}$ must be equal for all $\boldsymbol{k}$. Only if it is a vertex, i.e. if $\boldsymbol{G}^{\prime}(\boldsymbol{p}-\mathbf{0}) \neq \boldsymbol{G}^{\prime}(\boldsymbol{p}+\mathbf{0})$, the consistency criterion allows different values of $\boldsymbol{r}_{\boldsymbol{k}}$. Such a fact could be considered as natural if the graph's breakpoints are admitted as being exact reflections of the modeled situation. However, if one treats the breakpoint as a convenient idealization of smooth curves with rapidly changing slops, one has to demand the equality of all $r_{k}$ as it were the case with the smooth approximation of the demand curve. That is why such a case is considered distinctly in the definition of the interior equilibrium below, as it was done in [7].

Definition 2. The collection $\left(p, q_{1}, \ldots, q_{n}, v_{1}, \ldots, v_{n}\right)$ where $v_{k} \geq \mathbf{0}, \boldsymbol{i}=\mathbf{1}, \ldots, n$, is referred to as an interior equilibrium if, for the considered influence coefficients the collection $\left(\boldsymbol{p}, \boldsymbol{q}_{1}, \ldots, \boldsymbol{q}_{\boldsymbol{n}}\right)$ is an exterior equilibrium, and the consistency criterion is satisfied for all $\boldsymbol{k}$. Furthermore, if all $\boldsymbol{r}_{\boldsymbol{k}}$ in (4) have the same value, then the interior equilibrium is called strong.

The following theorem is an extension of Theorem 2 in [7].

Theorem 2. Under assumptions $\mathbf{A 1}, \mathbf{A 2}$ and $\mathbf{A 3}$, there exists a strong interior equilibrium.

\section{Demand Structure and Equilibrium}

This section examines how variations of the demand structure affect the equilibrium price and the aggregate production output. We are mostly interested not in quantitative variations but the qualitative ones predicted by the above introduced model. Although Theorems 1 and 2 of the existence of equilibrium are valid in the more general case of piecewise quadratic cost functions, to understand the analysis better, we will first consider pure quadratic cost functions, as in [7]. 
Hence, assume first that the cost functions have the form $\boldsymbol{f}_{\boldsymbol{i}}\left(\boldsymbol{q}_{i}\right)=\boldsymbol{c}_{\boldsymbol{i}} \boldsymbol{q}_{\boldsymbol{i}}+\boldsymbol{a}_{\boldsymbol{i}} \boldsymbol{q}_{\boldsymbol{i}}^{\mathbf{2} / \boldsymbol{2}}$ where $\boldsymbol{c}_{\boldsymbol{i}}>\mathbf{0}$ and $\boldsymbol{a}_{\boldsymbol{i}}>\mathbf{0}, \boldsymbol{i}=\mathbf{1}, \ldots, \boldsymbol{n}$. If one is only interested in the strong interior equilibrium then formula (4) from the consistency criterion can be rewritten as follows:

$$
v_{k}=\frac{1}{\sum_{i \neq k} \frac{1}{v_{i}+a_{i}}-r}, \quad k=1, \ldots, n
$$

where $r \in[-\infty, \mathbf{0}]$. If $r=-\infty$, then equation (5) has the unique solution $\boldsymbol{v}_{\boldsymbol{k}}=\mathbf{0}, \boldsymbol{i}=\mathbf{1}, \ldots, \boldsymbol{n}$. Otherwise, the following assertion is valid.

Lemma 1. ([7]). For any $r \in]-\infty, 0]$ there is a unique solution of equation (5) $\boldsymbol{v}_{\boldsymbol{k}}=\boldsymbol{v}_{\boldsymbol{k}}(\boldsymbol{r}), \boldsymbol{k}=\mathbf{1}, \ldots, \boldsymbol{n}$, that depend continuously upon $\boldsymbol{r}$. Furthermore, $\boldsymbol{v}_{\boldsymbol{k}}(\boldsymbol{r}) \rightarrow \mathbf{0}$ when $\boldsymbol{r} \rightarrow-\infty$, and $\boldsymbol{v}_{\boldsymbol{k}}(\boldsymbol{r})$ strictly increases up to $\boldsymbol{v}_{\boldsymbol{k}}(\mathbf{0})$ as $\boldsymbol{r}$ increases up to zero.

Equalities (2) for the quadratic functions give the linear dependences $q_{i}\left(\boldsymbol{p}, \boldsymbol{v}_{i}\right)=\frac{\boldsymbol{p}-\boldsymbol{c}_{\boldsymbol{i}}}{\boldsymbol{v}_{\boldsymbol{i}}+\boldsymbol{a}_{\boldsymbol{i}}}$. Thus for each $r \in[-\infty, 0]$ one can determine $v_{k}, \boldsymbol{i}=\mathbf{1}, \ldots, n$ from (5) and then construct the aggregate output function

$$
Q(p, r)=\sum_{i=1}^{n} \frac{p-c_{i}}{v_{i}+a_{i}}
$$

It is immediate that $Q(p, r)$ strictly increases by $\boldsymbol{p}$ and strictly decreases with respect to $\boldsymbol{r}$. Therefore the intersection points of the straight lines $G=Q(p, r)$ with the demand curve move steadily to the right as $r$ grows from $-\infty$ up to 0. The straight line $G=Q(p,-\infty)$ describes the dependence of the aggregate output upon the price $\boldsymbol{p}$ in case of the perfect competition, when the producers exclude the possibility of their individual influence on the price. On the other hand, the straight line $\boldsymbol{G}=\boldsymbol{Q}(\boldsymbol{p}, \mathbf{0})$ corresponds to the maximum (for the given cost functions) claims of the individual influence.

Now coming back to the piecewise quadratic cost functions we see that the picture is more complex. First, even if the influence coefficients are exogenous and fixed, the optimal response $\boldsymbol{q}_{\boldsymbol{i}}\left(\boldsymbol{p}, \boldsymbol{v}_{\boldsymbol{i}}\right)$ will not be a linear function as before but a piecewise linear function.

Example 2. Consider the piecewise quadratic cost function $\boldsymbol{f}$ from Example 1 and set $\boldsymbol{v}=\mathbf{0 . 5}$ as the agent's influence coefficient. Using the optimality response condition (2) one easily constructs the optimal response production function $q(p, v)$ as follows:

$$
q(p, v)=\left\{\begin{array}{lr}
0, & 0 \leq p \leq 1 ; \\
\frac{2}{3}(p-1), & 1<p \leq 2.5 \\
\frac{2}{5} p, & 2.5<p \leq 5 \\
\frac{2}{3}(p-2), & p>5
\end{array}\right.
$$

It is easy to see that the function in question is continuous and piecewise linear. 
However, the picture is even more complex if we want to restrict ourselves to the case of the optimal responses with consistent influence coefficients $\boldsymbol{v}_{\boldsymbol{i}}, \boldsymbol{i}=\mathbf{1 , 2}, \ldots, \boldsymbol{n}$. Here the optimal response production functions $\boldsymbol{q}_{\boldsymbol{i}}\left(\boldsymbol{p}, \boldsymbol{v}_{\boldsymbol{i}}\right)$, $\boldsymbol{i}=\mathbf{1}, \cdots, \boldsymbol{n}$, and hence their sum (collective response function) $\boldsymbol{Q}(\boldsymbol{p}, \boldsymbol{r})$ need not being continuous, as the consistent influence coefficients use to change their values with respect to the intervals over which the agents' cost functions do not change their second derivative. We illustrate that by the following example.

Example 3. Consider a particular case of our model with $\boldsymbol{n = 3}$ and the agents' cost functions given below:

$$
\begin{aligned}
& f_{1}\left(q_{1}\right)=\left\{\begin{array}{lc}
0.5 q_{1}^{2}+q_{1}, & 0 \leq q_{1} \leq 1 ; \\
0.4 q_{1}^{2}+1.2 q_{1}-0.1, & q_{1}>1 ;
\end{array} \quad f_{2}\left(q_{2}\right)= \begin{cases}q_{2}^{2}+2 q_{2}, & 0 \leq q_{2} \leq 1 ; \\
0.6 q_{2}^{2}+2.8 q_{2}-0.4, & q_{2}>1 ;\end{cases} \right. \\
& f_{3}\left(q_{3}\right)= \begin{cases}q_{3}^{2}+q_{3}, & 0 \leq q_{3} \leq 1 ; \\
0.75 q_{3}^{2}+1.5 q_{3}-0.25, & q_{3}>1 .\end{cases}
\end{aligned}
$$

If we set $r=-\mathbf{1 0}$ and restrict ourselves to the unit cube $\mathbf{0} \leq \boldsymbol{q}_{i} \leq \mathbf{1 , i}=\mathbf{1 , 2 , 3}$, then

$$
a_{1}=1, a_{2}=2, a_{3}=2
$$

Having substituted these parameters to the equations system (5) and solved it for the influence coefficients $v_{i}, i=1,2,3$, we obtain $v_{1}=\mathbf{0 . 0 9 1 3}, v_{2}=v_{3}=\mathbf{0 . 0 8 7 8}$. However, if for example, $q_{1}>1$ while $0 \leq q_{i} \leq \mathbf{1}, i=\mathbf{2 , 3}$, then

$$
a_{1}=0.8, a_{2}=2, a_{3}=2
$$

therefore $v_{1}=\mathbf{0 . 0 9 1 2 5}, v_{2}=v_{3}=\mathbf{0 . 0 8 6 1 9 7}$, which leads to a small "leap" of the optimal response production function $q_{1}\left(p, v_{1}\right)$ at the point $p_{1}=\mathbf{2 . 0 9 1 3}$; namely,

$$
\underset{p \rightarrow 2.0913-0}{\lim _{1}} q_{1}(p, 0.0913)=1 \text {, whereas } \lim _{p \rightarrow 2.0913+0} q_{1}(p, 0.09125)=1.0000561 .
$$

Similar discontinuities occur in the breakpoints of all other optimal response production functions leading to the discontinuities in the aggregate response function $Q(p, r)$.

Nevertheless, the principal qualitative behavior diagram does not change greatly if we consider the piecewise quadratic cost functions instead of purely quadratic ones. If we demand, in addition to assumptions $\mathbf{A 1}-\mathbf{A} 3$, that the second derivative $f^{\prime \prime}{ }_{i}\left(q_{i}\right)$ does not increase along with $\boldsymbol{q}_{i}$, then the only difference between the cases of pure and piecewise quadratic cost functions is that the continuous straight lines in the first case give place to piecewise linear graphs with breaks at a finite number of points, in the second case. Thus, in order to illustrate the qualitative phenomena clearer, below we follow [7] in the graphic representation of the demand and equilibrium behavior in case of pure quadratic cost functions.

First consider the situation of Fig. 1. Here the passive demand $\boldsymbol{G}(\boldsymbol{p})$ is zero if $\boldsymbol{p}>\boldsymbol{p}_{*}$ but equals $\boldsymbol{G}_{\mathbf{0}}$ when $\boldsymbol{p} \leq \boldsymbol{p}_{*}$, that is, there is a momentary leap of demand. The right-side limit $\boldsymbol{g}(\boldsymbol{p})$ here is zero if $\boldsymbol{p} \geq \boldsymbol{p}_{*}$ and equals $\boldsymbol{G}_{\mathbf{0}}$ when $\boldsymbol{p}<\boldsymbol{p}_{*}$. A positive active demand $\boldsymbol{D}_{\mathbf{0}}$ somewhat lifts the demand curve up. The straight lines (or piecewise linear graphs in case of piecewise quadratic cost functions) numbered $\mathbf{0}$ to $\mathbf{2}$ are the graphs of the aggregate production for various values of $\boldsymbol{r}$. More precisely, number $\mathbf{0}$ is attached to the case $\boldsymbol{r}=-\infty$, i.e. the 
perfect competition. Number $\mathbf{1}$ is assigned to the case $\boldsymbol{r}=\mathbf{0}$, whereas $\mathbf{2}$ is numbering the case of an intermediate value $\overline{\boldsymbol{r}}$ in which the aggregate production graph comprises the point $\left(\boldsymbol{p}_{*}, \boldsymbol{D}_{\mathbf{0}}+\boldsymbol{G}_{\mathbf{0}}\right)$. Because $\boldsymbol{G}^{\prime}\left(\boldsymbol{p}_{*}-\mathbf{0}\right)=\mathbf{0}$ and $\boldsymbol{G}^{\prime}\left(\boldsymbol{p}_{*}+\mathbf{0}\right)=-\infty$ at that point, then it represents an interior equilibrium. Another interior equilibrium is associated with the graph point $\left(p_{1}, D_{0}\right)$ where $G^{\prime}\left(p_{1}-\mathbf{0}\right)=G^{\prime}\left(p_{1}+\mathbf{0}\right)=\mathbf{0}$ and $r=\mathbf{0}$. Strictly speaking there is the third equilibrium in this situation, namely the point $\left(\boldsymbol{p}_{*}, \boldsymbol{D}_{\mathbf{0}}\right)$ which corresponds to some $\boldsymbol{r} \in(\overline{\boldsymbol{r}}, \mathbf{0})$, as in this point one has $\boldsymbol{G}^{\prime}(\boldsymbol{p}-\mathbf{0})=-\infty$ and $\boldsymbol{G}^{\prime}(\boldsymbol{p}+\mathbf{0})=\mathbf{0}$. However, the latter equilibrium is unstable: any growth of the active demand makes the price jump up to the value $p_{1}$.

Now let the economy have the state $\left(\boldsymbol{p}_{*}, \boldsymbol{D}_{\mathbf{0}}+\boldsymbol{G}_{\mathbf{0}}\right)$ and the active demand $\boldsymbol{D}$ starts growing up thus lifting up the demand curve. Until $\boldsymbol{D} \leq \boldsymbol{D}_{\mathbf{0}}+\boldsymbol{\delta}$ the situation keeps changing continuously with the price $\boldsymbol{p}_{*}$ kept intact and the demand $\boldsymbol{D}+\boldsymbol{G}_{\mathbf{0}}$ satisfied completely. The production increases on account of diminishing of the equilibrium influence coefficients (i.e. on account of $\boldsymbol{r}$ decreasing). When the breakpoint of the demand curve reaches line $\mathbf{0}$ (that corresponds to $\boldsymbol{D}=\boldsymbol{D}_{\mathbf{0}}+\boldsymbol{\delta}$ and to the perfect competition regime) then the price cannot be supported unchanged as $\boldsymbol{v}_{\boldsymbol{i}}=\mathbf{0}, \boldsymbol{r}=-\infty$, and they cannot be lowered anymore.

The next stage of the process is reflected in Fig. 2. If the active demand belongs to the interval $\left(D_{0}+\delta, D_{0}+G_{0}+\delta\right)$, then the equilibrium is represented by the point $\left(p_{*}, D_{0}+G_{0}+\delta\right)$ which corresponds to the perfect competition regime. Here neither price nor production output is affected by the active demand value, but a deficit appears because the aggregate demand $\boldsymbol{D}+\boldsymbol{G}_{\mathbf{0}}$ is not satisfied. Along with the active demand growing up to the value $\boldsymbol{D}_{\mathbf{0}}+\boldsymbol{G}_{\mathbf{0}}+\boldsymbol{\delta}$, the deficit will also go up to the passive demand value $\boldsymbol{G}_{\mathbf{0}}$.

The third stage comes with the active demand exceeding the value of $\boldsymbol{D}_{\mathbf{0}}+\boldsymbol{G}_{\mathbf{0}}+\boldsymbol{\delta}$. At this moment the equilibrium point $\left(\boldsymbol{p}_{*}, \boldsymbol{D}_{\mathbf{0}}+\boldsymbol{G}_{\mathbf{0}}+\boldsymbol{\delta}\right)$ loses stability, the price jumps to the value $\boldsymbol{p}^{*}>\boldsymbol{p}_{*}$ and the deficit vanishes due to the elimination of the passive demand. The economy jumps to a qualitatively new state. Although the aggregate production output has not fallen down, the prices are established on a level higher than the marginal costs. Further growing active demand will be satisfied completely on account of the corresponding growth of price.

Now suppose that the active demand starts going down. It is evident that the process will not be reversed. The equilibrium point will stay on line $\mathbf{1}$ until the active demand reaches the value $\boldsymbol{D}_{\mathbf{1}}<\boldsymbol{D}_{\mathbf{0}}$ and the price becomes equal to $\boldsymbol{p}_{*}$ but now at the lower production level than in the beginning of the process. Further falling active demand will force the economy to jump to the initial state: the price $\boldsymbol{p}_{*}$ being intact, the output volume will increase up to $\boldsymbol{G}_{\mathbf{0}}+\boldsymbol{D}_{\mathbf{1}}$ having satisfied both the active and passive demands completely. However, if the active demand returns only to its initial state $\boldsymbol{D}_{\mathbf{0}}$, the economy keeps staying at the equilibrium point $\left(\boldsymbol{p}_{\mathbf{1}}, \boldsymbol{D}_{\mathbf{0}}\right)$ of Fig. 1, the price will be fixed higher the critical price $\boldsymbol{p}_{*}$ for the passive demand, and only the active demand $\boldsymbol{D}_{\mathbf{0}}$ will be satisfied.

If the demand curve has not single but several steps, the behavior of prices will be similar. The only difference is in that the process will be repeated as many times as many steps there are to the right off line $\mathbf{0}$ in Fig. 1. Each leap of prices corresponds to a switch to a new regime, with an appropriate group of consumers abandoning the market and thus eliminating the deficit that had arisen before that.

Now consider the case when the active demand is stable but the passive demand structure changes on account of, say, a variation of the consumption ability of a part of consumers. We illustrate this process on an example of two groups of consumers in the absence of active demand. Again we restrict ourselves to an ideal case when the demand curve is a step function with two steps (Fig. 3). The price $p_{1}^{*}$ is critical for the poorer group of consumers with 
consumption volume $\boldsymbol{G}_{\mathbf{1}}$, whereas the price $\boldsymbol{p}_{\mathbf{2}}^{*}>\boldsymbol{p}_{\mathbf{1}}^{*}$ is such for the richer group with consumption volume $\boldsymbol{G}_{\mathbf{2}}$. The lines numbered by $\mathbf{0}$ and $\mathbf{1}$ have the same meaning as in the previous example, i.e. they are the aggregate production output graphs in the perfect competition regime and the maximum monopolistic tendencies regime, respectively. The intermediate graphs numbered as $\mathbf{2}$ and $\mathbf{3}$ are associated with two interior equilibria $\left(\boldsymbol{p}_{\mathbf{1}}^{*}, \boldsymbol{G}_{\mathbf{1}}+\boldsymbol{G}_{\mathbf{2}}\right)$ and $\left(p_{2}^{*}, G_{2}\right)$.

Now suppose that the consumption ability of the first (the poorer) group of consumers goes down. This process can be modeled by diminishing the critical price $p_{1}^{*}$. For simplicity purpose, we ignore a possible decrease of the consumption volume $\boldsymbol{G}_{\mathbf{1}}$. Again, the first stage of the considered process consists in lowering of the influence coefficients thus approaching the perfect competition regime.

This feature will be exposed by the market price going down with the production output unchanged and the aggregate demand satisfied. The price will be equal to the critical one for the poorer group of consumers. This will be taking place until the critical price reaches the level $p^{1}$ with the production switching to the perfect competition regime.

At the second stage when the critical price for the first group of consumers lies in the interval $\left(p_{\mathbf{0}}^{\mathbf{1}}, p^{\mathbf{1}}\right)$, the market price keeps going down along with the critical one, the perfect competition keeps being valid, but the aggregate production volume starts decreasing and a deficit arises (see Fig. 4). The deficit will increase as the price approaches the value $\boldsymbol{p}_{\mathbf{0}}^{\mathbf{1}}$. If the consumption ability keeps lowering then the market price jumps up to the level $\boldsymbol{p}_{2}^{*}$ ， and the economy makes a leap to the new equilibrium regime $\left(\boldsymbol{p}_{2}{ }^{*}, \boldsymbol{G}_{\mathbf{2}}\right)$. The poorer group of consumers abandons the market completely with the deficit eliminated.

If the consumption ability of the first group recovers, the process is not reversed completely (if no special extraneous efforts are made).

\section{Conclusion}

The qualitative analysis of the equilibrium behavior in dependence on the active demand level for the oligopolistic model with piecewise quadratic cost function is realized based upon that performed in [7] by V.A. Bulavsky for the case of pure quadratic cost functions. Introduction of this new form of cost functions keeps valid the previous results about existence and uniqueness of exterior equilibrium and existence of interior one. However, the situation is much more difficult in the new model, as the optimal response functions with consistent influence coefficients now need not be continuous.

In our forthcoming paper, we are going to examine the qualitative behavior of prices and production outputs for the case of piecewise linear cost functions. Such cost functions arise often when the model agents have several distinct production facilities among which they have to distribute the production volume so that the total cost be minimal.

\section{Acknowledgement}

This work was financially supported by the University of Nuevo Leon (UANL) Project PAICyT No. CA751-02 and by the Russian Foundation for Basic Research (RFBR), project No. 00-01-00404.

\section{References}

1. Ruffin R.J. Cournot oligopoly and competitive behavior. Rev. Econ. Studies. 1971. V. 38(4). N. 116. 
2. Sherali H.D., Soyster A.L. and Murphy F.H. Stackelberg -Nash-Cournot equilibria: characterizations and computations. Oper. Res. 1983. V. 31. N. 2.

3. Novshek W. On the existence of Cournot equilibrium. Rev. Econ. Studies. 1985. V. 52(1). N. 168.

4. Bulavsky, V.A., Kalashnikov, V.V. One-Parametric Method to Study an Equilibrium. Economics and Mathematical methods. 1994. V. 30. N. 4. (In Russian)

5. Bulavsky, V.A., Kalashnikov, V.V. Equilibrium in Generalized Cournot and Stackelberg Models. Economics and Mathematical methods . 1995. V. 31. N. 3. (In Russian)

6. Bulavsky, V.A. An Imagined Experiment in the Framework of the Generalized Cournot Model. Economics and Mathematical methods. 1996. V. 32. N. 2. (In Russian)

7. Bulavsky, V.A. Structure of Demand and Oligopolistic Equilibria. (Private communication) 
Notes 